\title{
Inhaltsverzeichnis
}

$1 \quad$ Einführung

Andrea Beyer, Britta Rathje

$\begin{array}{ll}\text { Lehren mit neuen Medien } & 5\end{array}$

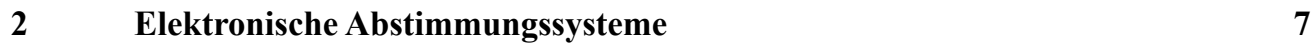

Florence De Boni

2.1 Was sind elektronische Abstimmungssysteme? ............................................... 7

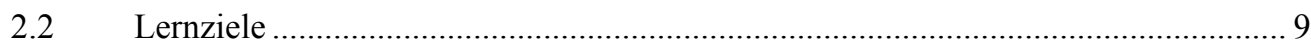

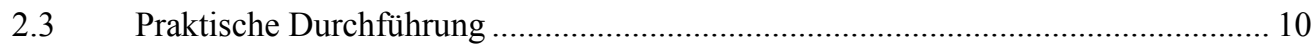

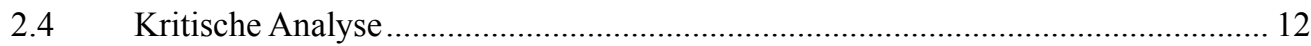

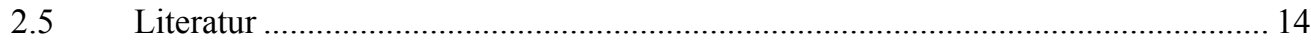

$3 \quad$ Einsatz von Blogs in der Lehre $\quad 15$

Petra Bauer und Silke Hamann

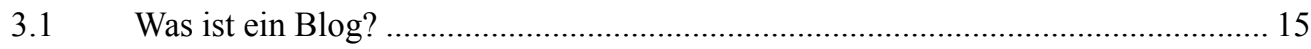

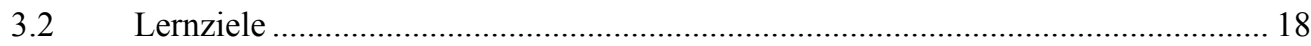

3.3 Durchführung: Lehr- und Lernszenarien ........................................................... 20

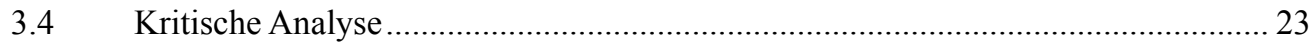

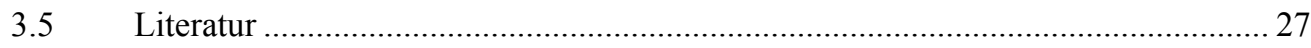

$4 \quad$ Einsatz von Tablets in der Lehre $\quad 29$

Anett Mehler-Bicher, Frank Mehler, Lothar Steiger

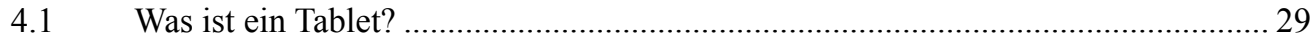

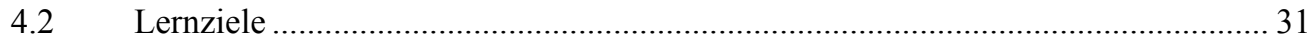

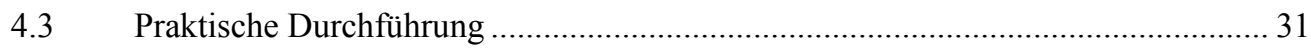

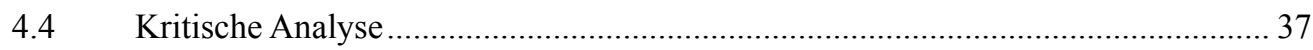

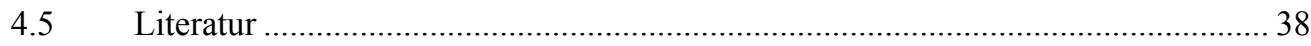

$5 \quad$ Podcasts $\quad 39$

Jens Reinhardt

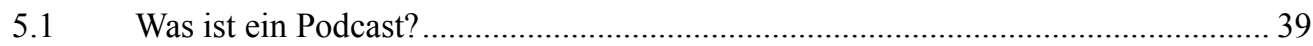

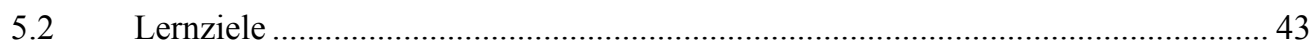




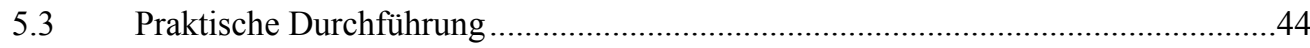

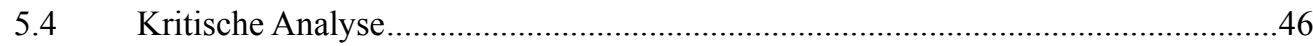

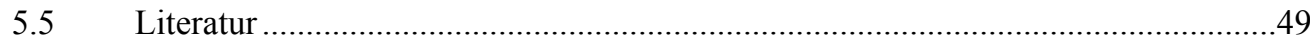

$\begin{array}{ll}\text { Aktivitätsorientierte Lehre } & 51\end{array}$

$6 \quad$ Rollenspiele $\quad 53$

Achim Saulheimer

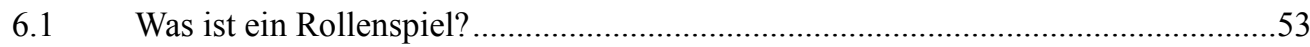

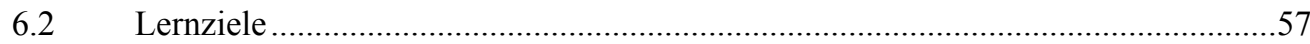

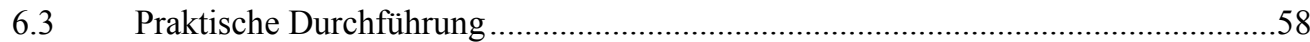

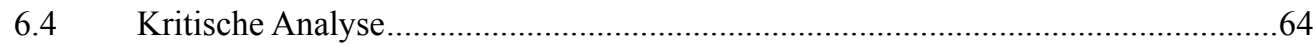

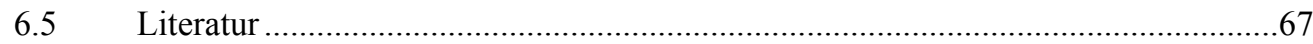

$7 \quad$ Unternehmenstheater als Ergänzung der Controlling-Ausbildung $\quad 69$

Ulrich Nissen

7.1 Was ist Unternehmenstheater, und wie lässt es sich in die ControllingAusbildung integrieren? .............................................................................6

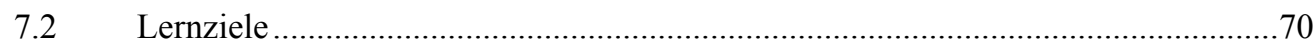

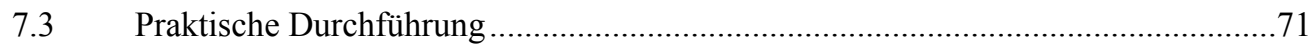

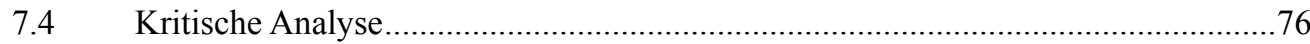

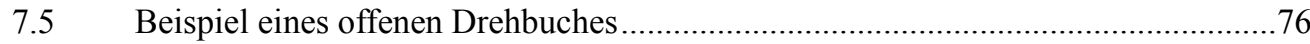

$8 \quad$ Das Controlling-Labor $\quad 87$

Carsten Wilken

8.1 Wie funktioniert das Controlling-Labor?.........................................................87

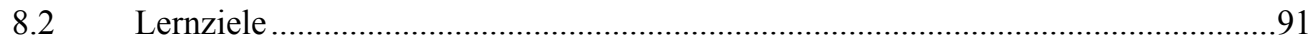

8.3 Praktische Durchführung .................................................................................... 94

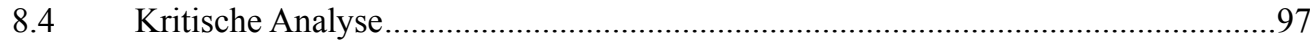

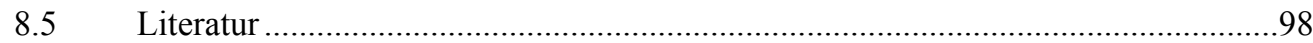

$9 \quad$ Gamification - Spielend leicht lernen $\quad 99$ Avo Schönbohm

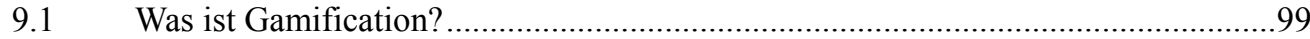

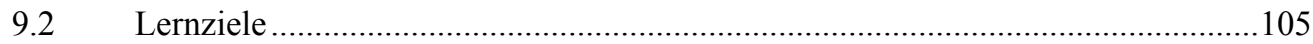

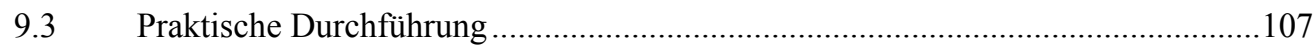

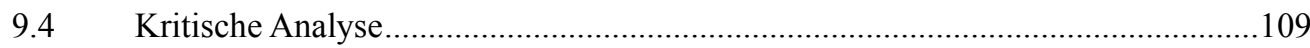

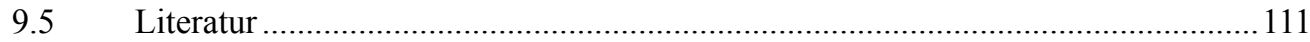


$10 \quad$ Speed Reading

Nicole Jekel

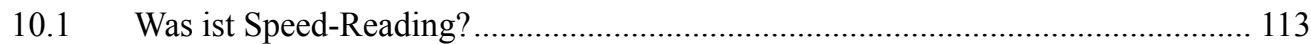

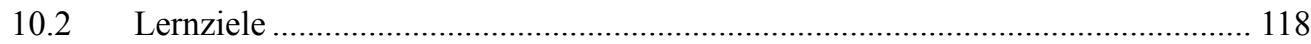

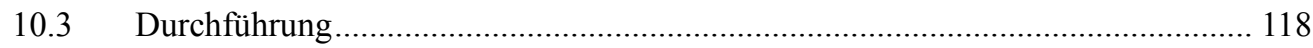

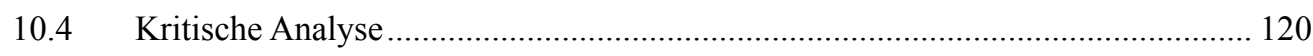

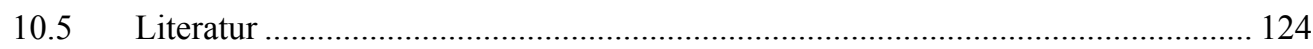

11 Intergeneratives Lernen - Mentoring einmal anders $\quad 125$ Kathrin Strässer-Knüttel

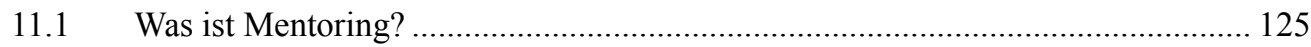

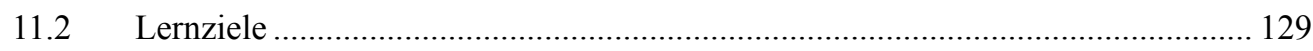

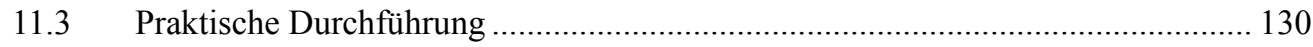

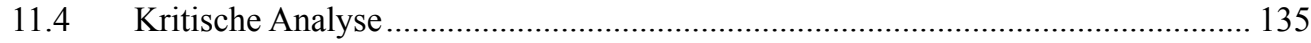

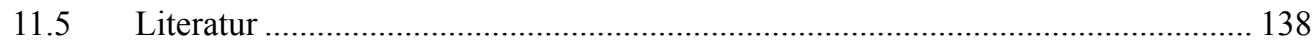

$12 \quad$ Soziale Formen des Lernens $\quad 139$

Kurt-Wolfgang Koeder

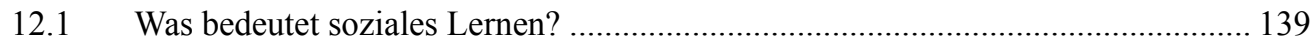

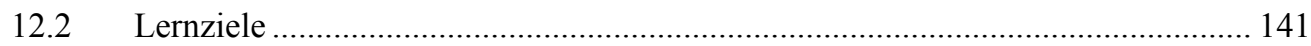

12.3 Praktische Durchführung ................................................................................ 142

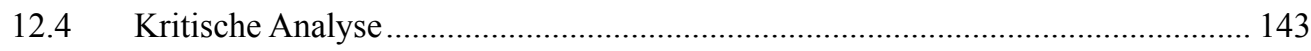

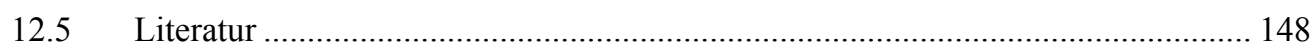

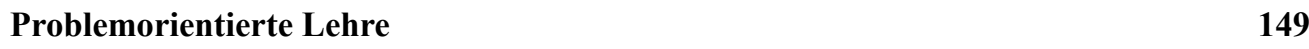

13 Fallstudien $\quad 151$

Herbert Paul

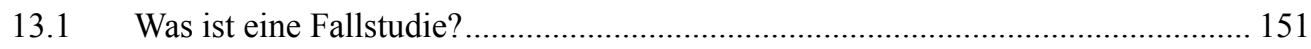

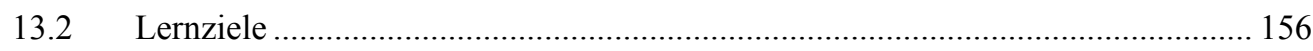

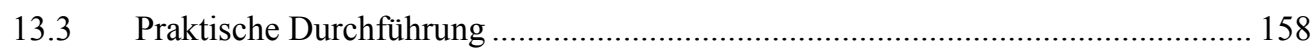

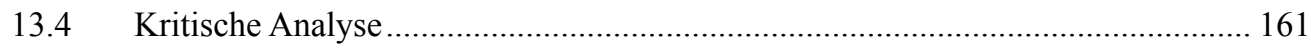

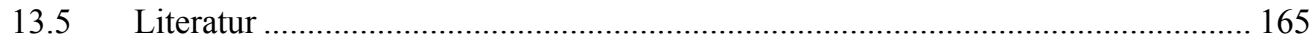

14 Projekte $\quad 167$

Ulrich Holzbaur, Carmen Venus

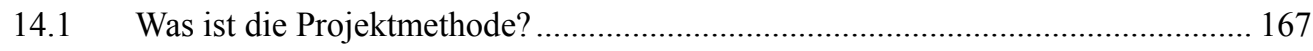

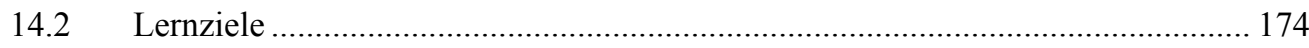




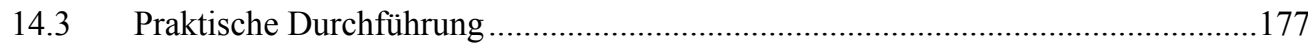

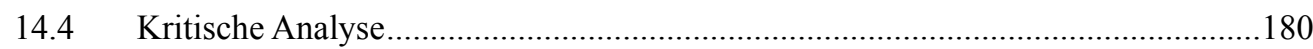

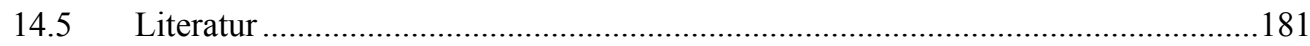

$15 \quad$ Studentisches Forschungsprojekt $\quad 183$ Ute Vanini

15.1 Forschendes Lernen durch studentische Forschungsprojekte ...............................183

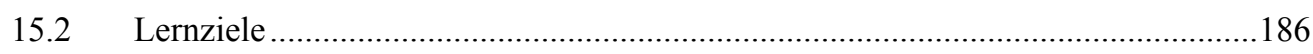

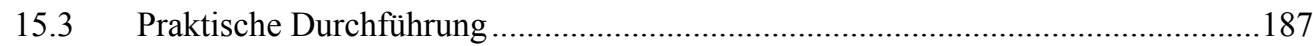

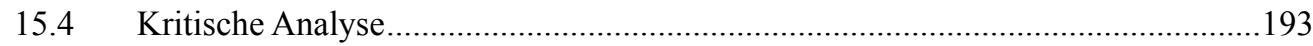

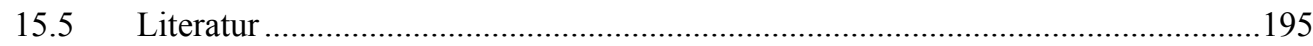

$16 \quad$ Lernende als Unternehmensberater $\quad 197$

Sven Fischbach und Britta Rathje

16.1 Was bedeutet „Lernende als Unternehmensberater“? ..........................................197

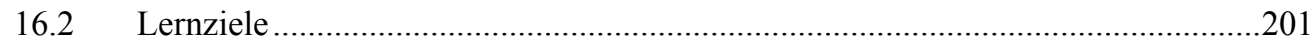

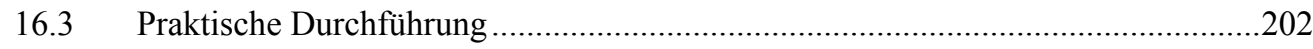

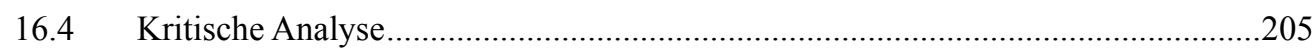

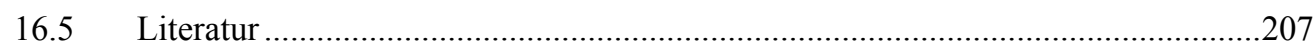

17 Unternehmensplanspiele $\quad 209$ Britta Rathje

17.1 Was ist ein Unternehmensplanspiel? ..............................................................209

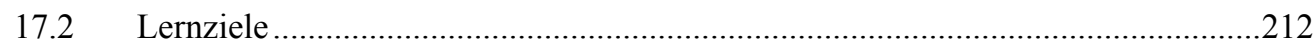

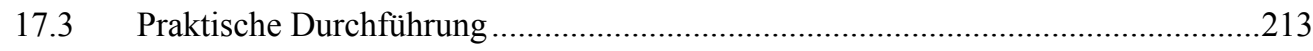

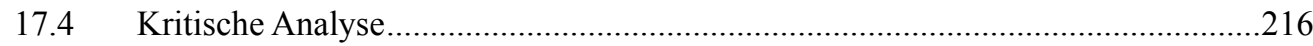

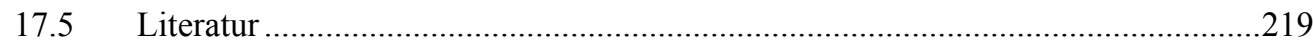

18 RealityPlanning $\quad 221$

Anett Mehler-Bicher, Michael Reiß, Jochen Schenscher, Lothar Steiger

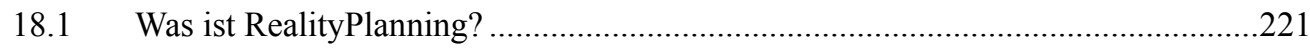

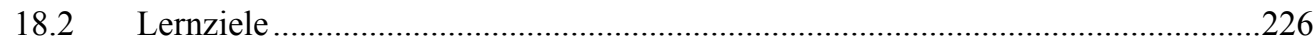

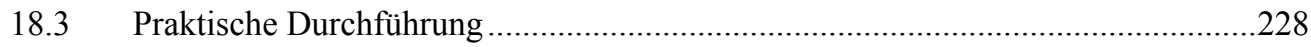

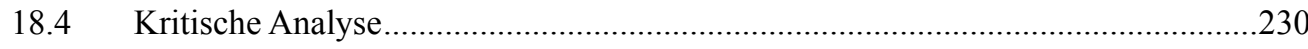


19 Durchlaufendes Veranschaulichungsbeispiel

Ulrich Nissen

19.1 Was ist ein durchlaufendes Veranschaulichungsbeispiel? ................................... 235

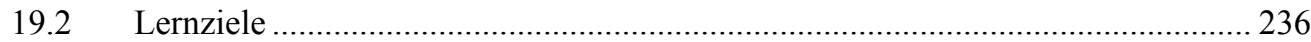

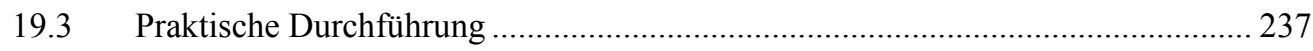

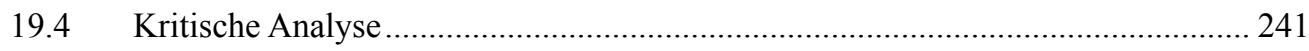

19.5 Beispielhafte Arbeitsmaterialien ...................................................................... 243

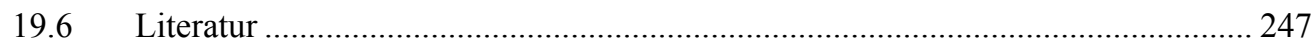

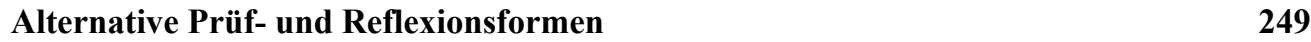

$20 \quad$ Bewusstes und reflexives Lernen $\quad 251$

Markus M. Böhner

$20.1 \quad$ Was ist bewusstes und reflexives Lernen? .................................................... 251

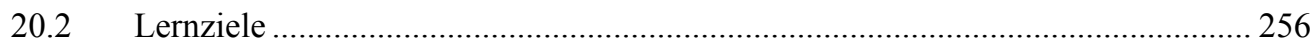

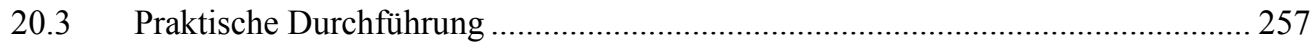

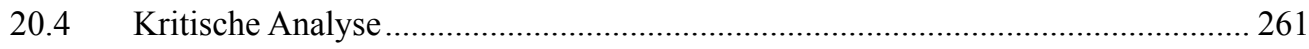

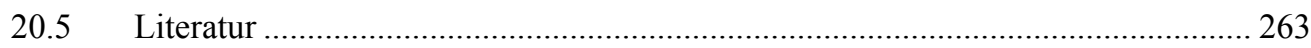

$21 \quad$ Buchbeitrag statt Klausur $\quad 265$ Andrea Beyer

21.1 Was ist der Leistungsnachweis?...................................................................... 265

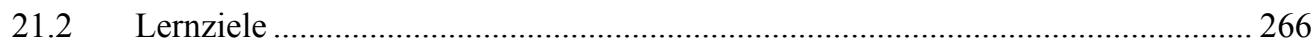

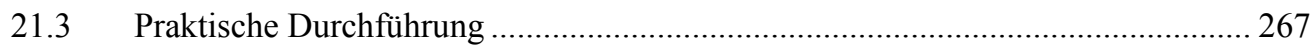

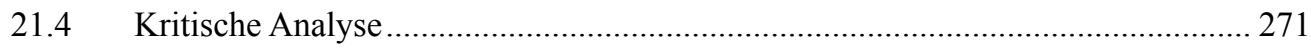

22 Rede und Debatte 273 Claudia Hoffmann

22.1 Was prüfen in Rede und Debatte? .................................................................... 273

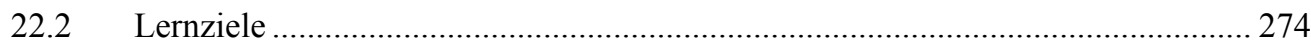

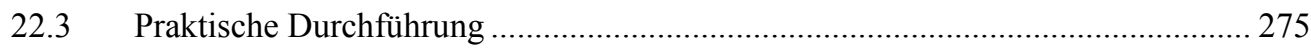

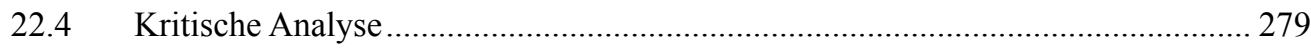

$\begin{array}{ll}\text { Autorenverzeichnis } & 283\end{array}$

$\begin{array}{lr}\text { Stichwortregister } & 287\end{array}$ 


\section{Gendering Hinweis}

Bei allen Bezeichnungen, die auf Personen bezogen sind, meint die gewählte Formulierung beide Geschlechter, auch wenn aus Gründen der leichteren Lesbarkeit die männliche Form steht.

\section{Bildnachweise}

(C) Stefan Merkle - Fotalia.com, Bild S. 1

(C) bloomua - Fotolia.com, Bild S. 29

(C) http://www.samsontech.com/site_media/legacy_docs/CO1U_Package_Img.jpg, Bild S. 39

(C) Elnur - Fotolia.com, Bild S. 53

(C) Elnur - Fotolia.com, Bild S. 69

(C) Eisenhans - Fotolia.com, Bild S. 87

(C) Michael Rosskothen - Fotolia.com, Bild S. 99

(C) Aaron Amat - Fotolia.com, Bild S. 113

(C) http://chronus.com/wordpress/wp-content/uploads/2012/10/reverse-mentoring.jpg, Bild S. 125

(C) adimas - Fotolia.com, S. 167

(C) DOC RABE Media - Fotolia.com, Bild S. 183

(C) jd-photodesign - Fotolia.com, Bild S. 197

(C) drubig-photo - Fotolia.com, Bild S. 221

(C) alphaspirit - Fotolia.com, Bild S. 235 